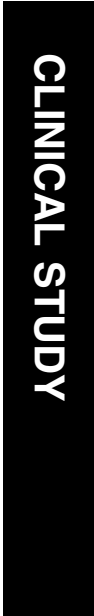 \\ Functional outcome and patient preferences following combined phaco-vitrectomy for macular hole without prone posturing
}

Hull and East Yorkshire Eye Hospital, Hull Royal Infirmary, Hull, North Humberside, UK

Correspondence: IM

Madgula,

9 Hartley Bridge,

Victoria Docks,

Hull HU9 1QG,

UK

Tel: +44 1482875875 ;

Fax: + 441482605331 .

E-mail: moorthyindira@

yahoo.co.uk

Received: 3 December 2006 Accepted in revised form: 19 March 2007

Published online: 13 April 2007

The authors have no financial interest in the work and have not received any funding for the same

\begin{abstract}
Background Macular hole surgery varies widely regarding the duration of gas tamponade, the use of patient posturing post-operatively, and whether or not cataract extraction is undertaken at the same time. Aim To analyse anatomical and functional success rate following macular hole surgery and to examine patient preferences regarding posturing and length of gas tamponade. Materials and methods Study design. Prospective, interventional and noncomparative case series.

Participants. Thirty patients with stage III and IV full-thickness macular hole who underwent macular hole repair during the period April 2005-January 2006.

Intervention. All eyes underwent a standard three-port pars plana vitrectomy, removal of posterior vitreous, internal limiting membrane (ILM) peel, C3F8 gas tamponade, and cataract extraction with IOL implantation. Patients did not posture post-operatively.

Outcome measures. Post-operative anatomic results, visual acuity, complications, patient preferences regarding surgical protocol, and subjective improvement in visual function. Results Patients were followed up postoperatively for 6 months. Primary anatomical hole closure was achieved in $96.7 \%$ eyes. Visual acuity improved in $83.8 \%$ patients. Two patients had raised intraocular pressure following surgery. A total of $96.9 \%$ of patients were happy with no posturing and a 2-month gas bubble.
\end{abstract}

IM Madgula and M Costen

Conclusions There is a high rate of anatomical and functional success in patients undergoing macular hole surgery without prone posturing. In our study, most patients preferred long-acting gas tamponade with no posturing over the option of posturing with short-acting gas tamponade.

Eye (2008) 22, 1050-1053; doi:10.1038/sj.eye.6702835; published online 13 April 2007

Keywords: macular hole; phaco-vitrectomy; posturing; patient preference

Introduction

Idiopathic full-thickness macular hole has an incidence of approximately 1 per 1000 population causing variable reduction of vision. ${ }^{1}$ Kelly and Wendel $^{2}$ performed the first successful vitrectomy for macular hole in 1991 and since then, both anatomical and functional success rates have continued to improve. Modifications to the original procedure include the use of different tamponade agents such as gas or silicone oil, use of adjuncts such as autologus serum and fibrin, transforming growth factor beta, platelets, thrombin, and peeling of the internal limiting membrane (ILM) ${ }^{2-5}$ Hole duration, stage, preoperative visual acuity, type of tamponade, or whether or not the ILM is peeled may be important predictors of success rate in macular hole surgery. ${ }^{5}$ Today, practice differs widely in relation to whether or not ILM peeling is routinely undertaken as part of the procedure, the duration of gas tamponade, the use of and 
duration of patient positioning post-operatively, and whether or not cataract extraction is undertaken at the same time.

\section{Aim of the study}

The aim of the study was to analyse anatomical and functional success rate following macular hole surgery combined with cataract surgery, but with no post-operative posturing, and to examine patient preferences regarding posturing and length of gas tamponade.

\section{Method}

The study consisted of a non-randomized, consecutive series of patients with idiopathic macular holes seen between April 2005 and January 2006. A total of 30 patients with macular hole were included in the study. Preoperative data collected included age, gender, best-corrected visual acuity (BCVA), refraction, lens status, duration and stage of macular hole, and the presence of posterior vitreous detachment (PVD) and any other associated retinal comorbidity. All patients underwent a thorough slit-lamp examination, dilated fundus examination, and an optical coherence tomographic assessment of the macula.

\section{Procedure}

After informed consent, all eyes underwent phacoemulsification with implantation of intraocular lens, followed by vitrectomy. PVD was induced where necessary, using active aspiration via the vitreous cutter over the optic disc. Once vitreous gel was cleared, ILM peeling was assisted with membrane blue visualisation. Peeling was completed in a rhexis fashion for approximately 1.5 disc diameters centred on the fovea. The periphery of the retina was examined for retinal breaks using scleral indentation, and any retinal breaks were treated with cryotherapy or argon laser photocoagulation. Fluid-air exchange was performed and the air was replaced with $16 \% \mathrm{C} 3 \mathrm{~F} 8$ gas. At the end of surgery, all patients received subconjunctival and topical antibiotics and steroids. Patients were seen at 1 day, 2 weeks, 2 months, and 6 months. Post-operatively, patients underwent visual acuity testing, measurement of intraocular pressure (IOP), and fundoscopy to assess the degree of gas fill and retinal integrity. Patients were not instructed to posture prone provided there was at least a $70 \%$ gas fill in the vitreous cavity on day 1 postoperative. All patients were however instructed to avoid lying supine. Anatomic success was defined as closure of the hole. Functional success was defined as an objective improvement in visual acuity. All patients were asked to fill in a patient satisfaction questionnaire at the end of
2 months. Data were analysed using an Excel spreadsheet and Snellen visual acuity was converted to the logarithm of the minimal angle of resolution $(\log$ MAR).

\section{Results}

\section{Patient demographics}

Thirty-one eyes of 30 (20 female, 10 male) consecutive Caucasian patients presenting with idiopathic, full-thickness macular hole were recruited in the study over a period of 10 months. The age of the patients ranged from 58 to 85 years with a mean age of $72.42 \pm 7.40$ (mean \pm SD). Average hole duration was 6.04 months; $64.5 \%$ holes were Stage III, $19.3 \%$ were stage II, and $16.1 \%$ were stage IV according to the staging criteria proposed by Gass. ${ }^{6}$ All patients were considered to have early to moderate cataract formation before surgery.

\section{Anatomical outcome}

After a mean followup period of 6 months, primary successful macular hole closure was achieved in $96.7 \%$ $(30 / 31)$ eyes. In one patient, the hole reopened after primary closure. Hole closure was achieved with repeat fluid gas exchange and facedown posturing for 10 days. Post-operatively, two patients had raised IOP that responded to medical treatment with a prostaglandin analogue. Peripheral retinal breaks were found in five eyes. These were treated with cryotherapy. There were no cases of endophthalmitis or retinal detachment in the current series.

\section{Functional outcome}

Post operatively, BCVA ranged from 20/200 to 20/30 (mean 20/63). Visual acuity improved by an average of two lines in $26(83.8 \%)$ eyes (Figure 1). In these eyes,

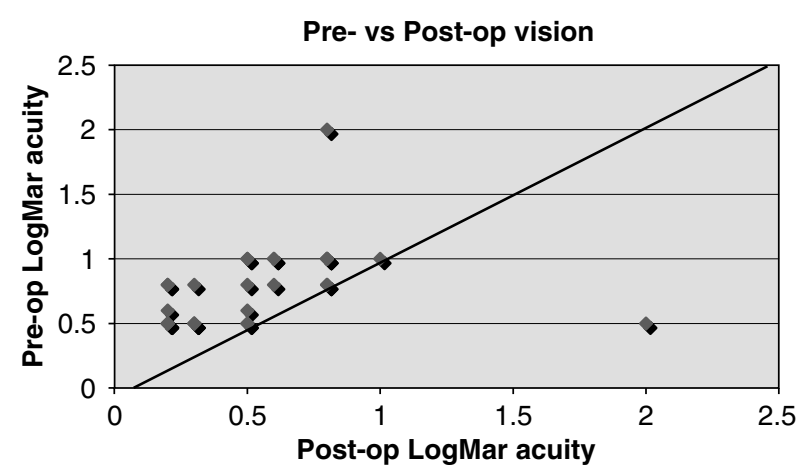

Figure 1 Chart demonstrating change in visual acuity from preoperative levels. (Snellen acuities have been converted to their $\log$ MAR equivalents). 
the BCVA ranged from 20/125 to 20/30 mean 20/60. In four $(12.9 \%)$ patients, the vision remained the same and worsened in one $(3.2 \%)$ eye. This was attributed to RPE atrophy.

\section{Patient satisfaction survey}

Patients were asked retrospectively via questionnaire if they were satisfied with their surgical protocol, or whether they would have preferred short-acting gas tamponade with at least 1 week face down posturing and cataract surgery at a later date. Twenty-nine (96.9\%) of the 30 patients preferred the protocol they received.

Five $(16.6 \%)$ of our patients reported difficulties relating to the gas tamponade. These included difficulty judging distances and feeling nauseous or unwell.

Twenty-seven $(90 \%)$ patients were extremely satisfied with the surgery and the aftercare that they received and were willing to undergo the procedure again.

\section{Discussion}

Studies of macular hole surgery are currently reporting anatomical success rates in excess of $90 \%$. There are several prognostic indicators for anatomical and functional success following surgery. These include stage of the hole, hole duration, hole size, preoperative visual acuity, and whether or not peeling of the ILM is performed. ${ }^{7-10}$ Various studies have confirmed that shorter hole duration and lower stage of macular hole, together with ILM peeling at the time of surgery are associated with a higher anatomical closure rate. ${ }^{8,9}$ However, the role of posturing in macular hole surgery remains controversial. A study designed to objectively evaluate the time of post-operative posturing by patients undergoing macular hole surgery questioned the importance of posturing in determining the success of macular hole surgery. ${ }^{11}$ Face down posturing is inconvenient, difficult, and unpopular, especially in the elderly. There have been reports of ulnar nerve palsies attributed to prolonged posturing. ${ }^{12}$ The current study confirms the high anatomical success rate of macular hole surgery. Our closure rate of $96.7 \%$ with an average hole duration of 6 months compares with other series reported in the literature. Jaycock et $a l^{8}$ have reported a closure rate of $95.2 \%$ for macular holes of less than 6 months duration and $91.7 \%$ for those where hole duration is between 6 and 12 months.

Our patient satisfaction survey underlined the fact that patients preferred to have long-acting gas tamponade rather than short-acting tamponade combined with posturing. However, it is important to note that patients were asked retrospectively to compare the treatment they received with a hypothetical situation and results of such a survey are likely to be biased.

Macular hole formation is more common in the elderly who may have other systemic problems affecting the ability to posture or compliance with the instructions. The 'Maculog' device designed by Verma et al ${ }^{11}$ to objectively measure posturing time showed that many patients were actually posturing less than half of the time they thought they were posturing. Based on these preliminary observations, they questioned the importance of posturing in determining the success of macular hole surgery. The current series found no adverse effects of not posturing, in terms of anatomical and functional outcome.

Visual acuity improved by an average of two lines in $83.8 \%$ of our patients with a mean visual acuity of $20 / 60$. In a series published by Tranos et al, ${ }^{1}$ only $50 \%$ of patients improved by two lines and $27 \%$ had a visual acuity of 20/40 or better. All the patients had C3F8 gas tamponade and were asked to posture face down for 1 week.

Nuclear sclerosis is one of the most common complications after macular hole surgery. This has been attributed to damage to the lens, harmful effects of intraocular irrigating solutions, and removal of the vitreous. ${ }^{13}$ Pre-existing lens opacities may impair visualisation of the ILM, thus hindering attempts to peel during surgery. ${ }^{14}$ Thus, the phacovitrectomy performed for the phakic patients in our series is cost effective and removes the need for subsequent surgery, allowing more rapid visual rehabilitation. Also phacoemulsification may be technically more challenging in a vitrectomised eye with a higher incidence of complications. Lack of vitreous support and previous damage to the posterior capsule and the zonular fibres have been suggested as increasing complication rates in vitrectomised eyes. ${ }^{8}$ The patient preference survey also indicated that patients were happy to undergo simultaneous cataract surgery, even with only early cataract formation, rather than undergo a second operation at a later date. Other authors have also advocated the use of phacovitrectomy without prone posturing for full-thickness macular holes. ${ }^{15}$ In our study, there were no significant complications associated with the surgery. Several patients had transient rises in IOP, which responded well to conservative treatment.

In addition to investigating the success rate of combined phacovitrectomy with ILM peel for macular holes, without the use of post-operative posturing, we also examined patient preferences regarding the surgical protocol. However, the study has its limitations. Patients were questioned regarding preference of surgical protocol retrospectively. This obviously induces potential bias to the responses. The technique outlined in this paper is the standard approach adopted by the surgeon (MC). Short-acting tamponade with prone posturing is 
offered to the patient if there are other patient factors involved, such as poor vision in the fellow eye, or difficulties with mobility, etc.

\section{Conclusions}

Patients in our study obtained a satisfactory anatomical and functional success rate after combined surgery without the use of prone posturing. There was no apparent adverse effect on anatomical or functional outcomes with this approach, when compared with reported success rates after posturing. Patients preferred the surgical protocol that involved phacovitrectomy and no posturing over the option to posture with shorter-acting gas tamponade. A randomised controlled trial of posturing $v$ s no posturing would be needed to better elucidate possible differences in outcomes for postured patients vs non-postured.

\section{Acknowledgements}

This study was presented at the Royal College Congress meeting, 2006 held at Manchester.

\section{References}

1 Tranos PG, Ghazi-Nouri SMS, Rubin GS. Visual function and subjective perception of visual ability after macular hole surgery. Am J Ophthalmol 2004; 138(6): 995-1002.

2 Kelly NE, Wendel RT. Vitreous surgery for idiopathic macular holes. Results of a pilot study. Arch Ophthalmol 1991; 109: 654-659.
3 Wender J, Iida T, Lucian A. Morphologic analysis of stage 3 and stage 4 macular holes: implications for treatment. Am J Ophthalmol 2005; 39: 1-10.

4 Gregor ZJ. Macular holes viewed objectively. Br J Ophthalmol 1997; 81(2): 98-99.

$5 \mathrm{Oz}$ O, Fudemberg SJ, Cakir B. Predictors of success in macular hole surgery with emphasis on the internal limiting membrane (ILM) and ILM peeling. Ophthalmic Surg Lasers Imaging 2004; 35(3): 207-215.

6 Johnson RN, Gass JD. Idiopathic macular hole. Observation, stages of formation and implications for surgical intervention. Ophthalmology 1988; 95(7): 917-924.

7 Kang S W, Ahn K, Ham D-I. Types of macular hole closure and their clinical implications. Br J Ophthalmol 2003; 87(8): 1015.

8 Jaycock PD, Bunce C, Xing W. Outcomes of macular hole surgery: implications for surgical management and clinical governance. Eye 2005; 19: 879-884.

9 Kumagai K, Furukawa M, Ogino N. Vitreous surgery with and without internal limiting membrane peeling for macular hole repair. Retina 2004; 24: 721-724.

10 Ullrich S, Haritoglou C, Gass C. Macular hole size as a prognostic factor in macular hole surgery. $\mathrm{Br} J$ Ophthalmol 2002; 86(4): 390.

11 Verma D, Jalabi MW, Watts WG. Evaluation of posturing in macular hole surgery. Eye 2002; 16: 701-704.

12 Salam A, Harrington P, Raj A. Bilateral ulnar nerve palsies, an unusual complication of posturing after macular hole surgery. Eye 2004; 18(1): 95-97.

13 Sawa M, Saito Y, Hayashi A. Assessment of nuclear sclerosis after nonvitrectomizing vitreous surgery. Am J Ophthalmol 2001; 132(3): 356-362.

14 Kotecha AV, Sinclair S H, Gupta A K. Pars plana vitrectomy for macular holes combined with cataract extraction and lens implantation. Ophthalmic Surg Lasers 2000; 31: 387-393.

15 Simcock PR, Scalia S. Phacovitrectomy without prone posturing for full thickness macular holes. Br J Ophthalmol 2001; 85(11): 1316-1319. 